innate conservatism of mankind and one wonders whether the Romans were faced by a similarly militant Celtic environmental lobby when first they dug the ditches.

\section{The EISCAT project} from G. N. Taylor

THE recent anouncement that the UK Science Research Council is joining EISCAT draws attention to some significant current developments in upper atmospheric research. EISCAT stands for the European Incoherent Scatter Organisation, an association of research organisations from six countries which has been set up to build and operate a large new radar system in the auroral region of Northern Scandinavia. In this case the capital and running costs, and overall control of the project, are being shared between Research Councils or similar bodies in Britain, France, Finland, West Germany, Norway and Sweden. After preparatory discussions and technical studies lasting several years it has been decided to base the main technical centre at Troms $\varnothing$ in Norway, and the administrative headquarters at Kiruna in Sweden. The organisation will be governed by a Council made up of representatives from all the parent bodies, and Professor Tor Hagfors from the Technical University of Trondheim has been appointed as the first Director of the facility.

The incoherent scatter technique originated from a proposal by W. E. Gordon in 1958, for building a very large radar system in order to detect weak Thomson scattering from thermal electrons in the ionosphere. Originally conceived as a way of measuring electron density and temperature in the upper ionosphere from the ground, the method has developed into one of the leading diagnostic tools not only for the upper atmospheric plasma but also for thermonuclear plasmas. From experimental work done in the 1960 s, it was soon discovered that both electron and positive ion temperatures could be measured independently, and later that plasma drift speeds and fluxes of superthermal electrons could also be determined. From these direct measurements, which are made as functions of height and time, many other physical parameters can be derived indirectly: among the most important are the electrostatic field strength, the temperature and wind speed of the neutral air, and energy fluxes. In collaboration with other ground-based techniques, or with rocket or satellite-borne instruments, incoherent scatter can give remarkably complete information about physical processes and conditions in the upper atmosphere. Incoherent scatter does not however supersede satellite experiments, even though some of the parameters measured are the same. For complete understanding, the upper atmosphere must be seen as a threedimensional time-varying medium: the radar works in height and time, whereas most satellite measurements are expressed as functions of latitude and longitude.

Incoherent scatter radars are complicated and expensive, requiring very high power transmitters, large antennae and sophisticated data processing equipment. Until now, no more than seven stations world-wide have ever been operating at the same time, and most of these have been at middle latitudes. The EISCAT facility will be only the second station in either of the auroral zones, which are the key regions for observing interactions between the ionosphere, the magnetosphere, the neutral atmosphere and the interplanetary plasma, and where many important and poorly understood phenomena occur. The radar should come into operation in 1978 or 1979 , and will then be operated intensively for at least a decade, with its time shared between a routine synoptic programme and special experiments by scientists from the participating countries. In Britain, research groups from a number of Universities and from the SRC's Appleton Laboratory are planning to use the facility. The scientific scope for European workers will be enhanced by the proximity of two rocket launching sites, and of many other ground-based instruments, at well established upper atmospheric observatories in adjacent areas

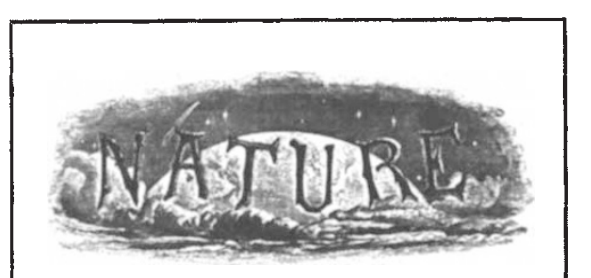

\section{A hundred years ago}

THE first general meeting of the Mineralogical Society of Great Britain and Ireland is held to-day at the Scientific Club, Saville Row, at 12 o'clock (noon), when the chair will be taken by Mr. H. C. Sorby, F.R.S. The first ordinary meeting will be held at the same place and time to-morrow, when a paper will be read on the Scottish Rhombohedral Carbonates, by Prof. M. Forster Heddle, M.D.

from Nature, 13, February 3, 274; 1876 of each of the three Scandinavian countries. A wide variety of other data will thus be available to complement and enhance EISCAT observations.

In contrast to most of the earlier incoherent scatter sites, the EISCAT system will be designed solely for upper atmospheric research. It will be unusual in having two radars on widely different frequencies. One in the VHF band (at $224 \mathrm{MHz}$ ) will be a conventional monostatic type, designed to achieve the maximum sensitivity, and to explore the highest and lowest regions. The other will operate in the UHF band (at $933 \mathrm{MHz}$ ), and will be a multistatic radar of the type pioneered in France, and also used in Britain, capable of determining the true vector plasma drift velocity. The transmitters for both radars will be at Troms $\varnothing$ : the VHF receiver and one UHF receiver will be co-sited with the transmitters, while remote UHF receiving stations will be built at Kiruna and at Sodankylä, in Finland, respectively 200 and $400 \mathrm{~km}$ distant from Troms $\varnothing$. Both radars will be designed with very flexible modes of transmission modulation, which should allow for a wide variety of experiments to be conducted. Comprehensive on-line and off-line data processing equipment will also be provided to obtain the maximum possible rates of useful information, and to monitor the progress of observations in real time. These features, together with the uniquely favourable geographical position of the site, should allow European scientists to make significant new contributions to an important field of geophysics.

\section{Bacterial chemotaxis and methionine}

from John S. Parkinson

Nearly a century ago, Engelmann, Pfeffer and others showed that bacteria are attracted or repelled by various chemicals. Today studies on chemotactic behaviour in Escherichia coli and other bacteria have begun to reveal molecular mechanisms of sensory transduction and motility in microorganisms. The central machinery of chemotaxis is still, however, very much a mystery. Several recent reports suggest that the amino acid methionine may provide the biochemical key to understanding chemotactic behaviour in $E$. coli.

The methionine requirement for chemotaxis is a specific and continuous one. $E$, coli mutants that are unable to synthesise methionine become nonchemotactic when deprived of exogenous methionine and chemotactic ability returns upon restoration of this amino 